\title{
Introduction to Selected Papers Based on Invited Talks Presented on the 18th World Conference on Non-destructive Testing
}

\author{
Jasson Gryzagoridis
}

Published online: 21 November 2012

(C) Springer Science+Business Media New York 2012

The 18th World Conference on Non-destructive Testing (WCNDT) was hosted by South Africa in the International Conference Centre at the coastal city of Durban during 16-20 April of 2012. It was the first time in the history of WCNDT that it came to Africa and from my perspective it ranks amongst the most impressive past conferences in terms of venue, organisation, technical content, hospitality and entertainment.

WCNDT takes place every four years and showcases innovation and state-of-the-art in NDT. This year's theme "NDT IN SERVICE OF SOCIETY" (in the words of Mike Farley, the Chairman of the International Committee for Non-destructive Testing) reminded us of why our discipline is so important and why we need to share our knowledge, technology and experience to the benefit of all. One of the highlights of the Conference was the speech by Professor Harold Groto, Nobel Prize Winner for Chemistry in 1996, with a message on research as a stimulus to development of technologies and applications.

The 5 keynote speakers and 25 invited overview speakers together with more then 500 talks in 8 parallel sessions mostly devoted their time to addressing the current needs of non-destructive testing methods to obtain information not just on the presence of a flaw but in addition on its location, type, orientation and size.

The industrial exhibition that always accompanies the Conference displayed the traditional Technological Arsenal of the discipline with very few innovations. Yet again it was apparent that innovation in NDT is slow; on average, it takes $35^{+}$years to mature a new technology from inception to a

J. Gryzagoridis $(\bowtie)$

Department of Mechanical Engineering, University of Cape

Town, Rondebosch 7701, South Africa

e-mail: Jasson.Gryzagoridis@uct.ac.za commercial accomplishment. This is really not surprising when one considers that in general industries are very mindful of safety and therefore innately conservative and will not easily run the risk of replacing an established traditional NDT method which in addition is safeguarded by codes and standards.

The 25 invited speakers of the Conference were given the choice of submitting a manuscript of their talk for possible publication in this journal which, as an outsider I can attest, enjoys a reputation of eminence in the NDT fraternity for the reliability and excellence of the material that appears in its issues. About a third of the invited speakers chose this option and subsequent to peer review of the manuscripts, JONE takes pleasure in presenting the accepted papers in the current issue. A brief description of the individual papers follows.

Trends and Stakes of NDT Simulation-by Pierre Calmon This is a review paper that also covers some current trends and perspectives. The author presents trends in NDT modelling where FEM is used for benchmarking and the emphasis is put on fast semi-analytical (integral) methods.

Synthetic Aperture Focusing and Time-of-Flight Diffraction Ultrasonic Imaging-Past and Present-by Martin Spies et al. The paper provides and overview of the investigations concerned with SAFT and TOFD that have been conducted for the past 20 to 30 years. The review is illustrated with a number of practical results and shows some of the imaging tradeoffs for a number of algorithms that one might chose from to refine the characterisation of an indication.

Guided Wave Inspection and Monitoring of Railway Trackby Philip Loveday This is a review paper that summarises the current state of the art for guided wave inspection of 
rail. The paper also provides some very appropriate critical thinking on previous results, as well as future opportunities and informs of the exciting research potential in this area.

Model-Based Guided Wave NDE: The Evolution of Guided Wave NDE from "Magic" to "Physically Based Engineering Tool"-by Younho Cho An overview is presented of various topics in the area of guided waves that have analytical tools as the basis. Some of the examples shown are guided wave modal analysis with dispersion curves, guided wave scattering models, and nonlinear guided wave feature analysis for micro-damage detection.

Laser Based Nondestructive Inspection Techniques-by Jasson Gryzagoridis The paper presents an overview of emerging laser-based optical interferometric NDT techniques, such as Holographic Interferometry, ESPI and Shearography. It focuses on the existing few reports on NDT performed in the industrial arena by researchers using custom-built proprietary inspection equipment.

Guest Editor 